\title{
The effect of transcatheter aortic valve implantation on intraoperative left ventricular myocardial performance index
}

\author{
Takashi Ota, Kosaku Toyota, Yasuhiro Koide
}

Department of Anaesthesia Shonan Kamakura General Hospital

Myocardial Performance Index $=(\mathrm{a}-\mathrm{b}) / \mathrm{b}=(\mathrm{ICT}+\mathrm{IRT}) / \mathrm{ET}$

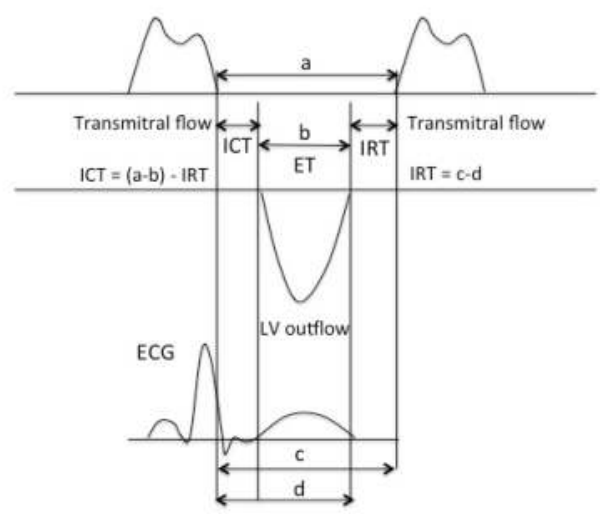

\section{Background and Goal of Study:}

Transcatheter aortic valve implantation (TAVI) procedure for patients with aortic stenosis is expected to reduce left ventricular after load and following improvement of cardiac function.

However, the effect of TAVI on intraoperative left ventricular global function including diastolic function gets less attention.

Myocardial performance index (MPI) is an index that incorporates both systolic and diastolic time intervals in expressing global systolic and diastolic ventricular function.

This retrospective observational study is aimed to investigate the intraoperative left ventricular MPI to assess the effect of TAVI on left ventricular global function.

\section{Materials and Methods}

The study was approved by Institutional Review Board of Shonan

Kamakura General Hospital (November, 2015).

Subjects: Patients who underwent TAVI procedure using

transfemoral approach with a balloon-expandable valve under general anesthesia

Study duration: June Ist $2014 \sim$ July 3 Ist 2015

Exclusion criteria:

Cases with severe intraoperative complications which were needed for circulatory support devices.

Cases which needed post dilatation for moderate to severe AR after deployment.

Time point of Measurements:

Before aortic balloon valvuloplasty and after prosthetic valve deployment

I I patients who underwent transfemoral TAVI procedure using SAPIEN XT valve under general anaesthesia were recruited in this study.

Ejection time (ET), isovolumic contraction time (ICT), and isovolumic relaxation time (IRT) were measured from pulse wave tissue Doppler waveforms of mitral annulus obtained using TEE during TAVI procedure. MPI was calculated as (ICT+IRT)/ET.

The MPI and Stroke volume before balloon aortic valvuloplasty and after prosthetic valve deployment were compared using paired t-test. Statistical significance was defined as the $P$ value less than 0.05 .

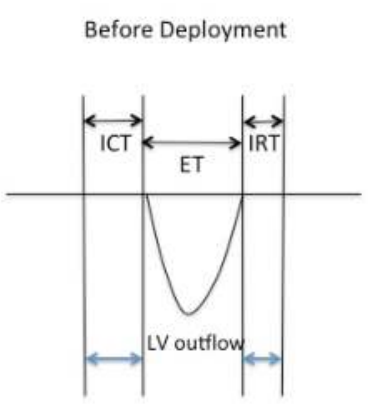

$\mathrm{MPI}=0.60 \pm 0.16$

$\mathrm{ICT} / \mathrm{IRT}=1.48 \pm 0.66$
After Deployment

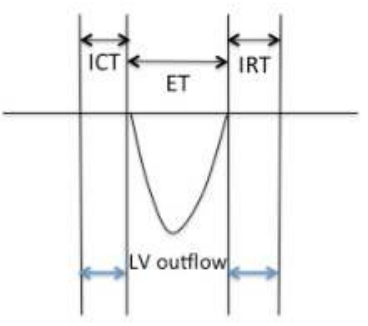

$\mathrm{MPI}=0.62 \pm 0.15$

$\mathrm{ICT} / \mathrm{IRT}=1.13 \pm 0.54$

\section{Results:}

The MPI before balloon aortic valvuloplasty and after prosthetic valve deployment were $0.60 \pm 0.16$ and $0.62 \pm 0.15$, respectively. There were no significant differences.

ICT to IRT ratio before balloon aortic valvuloplasty was $1.48 \pm$ 0.66 , and significantly decreased to $\mathrm{I} . \mathrm{I} 3 \pm 0.54$ after prosthetic valve deployment.

\section{Discussion:}

These results of this study show us two things.

First, TAVI procedure might not affect to left ventricular global function. According to our previous study, TAVI procedure shows little increasing of LVEDP after deployment but does not show worsening of E/e'. We suppose this increasing of LVEDP is caused by Myocardial ischemia in micro-circulation. No significant change of MPI supports TAVI procedure preserve left ventricular global function despite of the increasing of LVEDP.

Second, the decrease of ICT to IRT ratio might show the reduction of left ventricular afterload. Due to severe AS, Aortic valve motion is restricted. This immobility needs high threshold pressure, and it results to prolonged ICT. As TAVI procedure reduce left ventricular afterload, this prolongation will be corrected. How the decrease of ICT to IRT ratio affect to left ventricular global function is unknown, but the reduction of left ventricular afterload could contribute to preservation of left ventricular global function despite of the increasing of LVEDP. Further study will be needed to reveal this question.

\section{Conclusion:}

TAVI procedure does not affect to intraoperative left ventricular myocardial performance index but slides LV outflow time phase just after prosthetic valve implantation. 